\title{
Detailed-balance efficiency limits of two-terminal perovskite/silicon tandem solar cells with planar and Lambertian spectral splitters
}

\author{
Verena Neder, ${ }^{\text {a,b }}$ Stefan W. Tabernig $\odot,{ }^{b}$ and Albert Polman $\oplus^{b, *}$ \\ ${ }^{a}$ University of Amsterdam, Institute of Physics, Amsterdam, The Netherlands \\ ${ }^{b}$ NWO-Institute AMOLF, Center for Nanophotonics, Amsterdam, The Netherlands
}

\begin{abstract}
We derive the photovoltaic conversion efficiency limit for two-terminal tandem solar cells with a perovskite top cell and silicon bottom cell with an embedded spectrum splitter. For large-bandgap top-cells, a spectrum splitter strongly enhances the efficiency because of enhanced light absorption and trapping. A Lambertian spectral splitter shows a significantly improved effect compared with a planar splitter. We find an ideal efficiency enhancement for a 500-nm thick top cell of $6 \%$ absolute for bandgaps above $1.75 \mathrm{eV}$. Vice versa, the use of a spectral splitter geometry enables the use of a thinner top cell. Using experimental parameters for perovskite cells, we show that for a top-cell bandgap of $1.77 \mathrm{eV}$ a $2.7 \%$ absolute efficiency enhancement can be achieved. The calculations in this work show that integration of a spectral splitter into perovskite/silicon tandem cells for which the top cell is limiting the overall current can lead to a large increase in efficiency, even with realistic experimental losses and nonunity reflection of the spectral splitter. () The Authors. Published by SPIE under a Creative Commons Attribution 4.0 International License. Distribution or reproduction of this work in whole or in part requires full attribution of the original publication, including its DOI. [DOI: 10.1117/1.JPE.12.015502]
\end{abstract}

Keywords: tandem solar cells; detailed balance limit; spectral splitting; Lambertian light trapping; perovskite.

Paper 21085G received Oct. 19, 2021; accepted for publication Jan. 28, 2022; published online Feb. 16, 2022.

\section{Introduction}

At present, solar photovoltaics (PV) has an installed capacity of around $600 \mathrm{GW}_{\mathrm{p}}$ worldwide. ${ }^{1}$ Following the IRENA roadmap, by $2050 \mathrm{PV}$ should reach an installed capacity of $8.5 \mathrm{TW}_{\mathrm{p}}$ to account for $43 \%$ of the total installed power capacity for electricity generation. ${ }^{2}$ With the costs of the cells determining only a small part of the costs of a PV system, raising their efficiency is a key method to reduce the cost of PV per $\mathrm{kW}_{\mathrm{p}}$. Also, higher-efficiency panels take up less space, which is essential as PV is applied at very large scale. Therefore, in PV research, it is crucial to fight for every digit that can be gained in cell efficiency. With $95 \%$ of the total production in 2019, the market is strongly dominated by single-junction Si-wafer-based PV technology. ${ }^{3}$ Silicon-based tandem solar cells have the potential to raise the efficiency beyond the theoretical limit of $29.4 \%$ for Si-only cells. ${ }^{4}$ The combination of perovskite and $\mathrm{Si}$ in a 2-terminal (2T) or 4-terminal (4T) tandem configuration is one of the most growing and promising concepts. Recently, a Si-perovskite $2 \mathrm{~T}$ tandem solar cell was presented with an efficiency of $29.15 \% \%^{5}$ well above the record for a single-junction Si cell of $26.7 \% .^{6}$

One aspect that is of high importance to improve the performance of tandem cells is light management to optimize the coupling and distribution of sunlight in the tandem subcells. ${ }^{7-10}$ Recent work has focused on minimizing reflection from the top or interlayers ${ }^{11-14}$ and reducing parasitic absorption in the inactive layers, such as transparent conductive layers, ${ }^{9,15,16}$ and to optimize light trapping in the top and bottom cells. ${ }^{17,18}$ A concept that has not been studied in much detail is to control the spectral splitting of light directed into the two subcells. ${ }^{19}$ We note that other light management strategies to enhance efficiency such as enhanced light

*Address all correspondence to Albert Polman, a.polman@amolf.nl 
incoupling and backreflection from the bottom cell are also relevant; they are not discussed here. ${ }^{20}$ Spectral splitting can be achieved by an additional interlayer between the top and bottom cells that effectively reflects the part of the spectrum with energy above the bandgap of the top cell, and that transmits the remainder to the bottom cell. In an ideal case, the low- and highenergy spectral bands are fully split between the cells, so maximum current and voltage can be harvested. However, in practical geometries, a low-energy tail close to the top-cell bandgap is always transmitted due to incomplete light absorption in the top cell and is then absorbed in the underlying cell [Fig. 1(a)]. This transmitted tail creates higher thermalization losses in the bottom cell and should thus be avoided.

Earlier, spectral splitting in $4 \mathrm{~T}$ tandem cells has been modeled, ${ }^{19}$ and it was predicted that, by integrating a spectral splitting light trapping layer, an efficiency gain between $0.5 \%$ and $3 \%$ (absolute) and a two- to threefold reduction in thickness of the top cell can be reached, depending on the diffusion length of the absorber material. The benefits of spectral splitting in $2 \mathrm{~T}$ tandem concepts have been studied previously to achieve current matching between top and bottom cells, using Bragg reflectors ${ }^{22-25}$ or three-dimensional photonic crystals ${ }^{26-28}$ as intermediate layers, and small spectrum splitting improvements for Si-based top and bottom cells could be shown. Current matching is a limiting factor in $2 \mathrm{~T}$ perovskite/Si tandems for perovskites with a bandgap below $E_{\mathrm{BG}}=1.73 \mathrm{eV}$. In that case, the current generated in the perovskite can exceed that in the $\mathrm{Si}$, depending on the absorption in the top cell, in which case no spectral splitter is needed. However, a spectral splitter enables the use of a smaller perovskite cell thickness to obtain current matching with low-gap perovskites. This can already lead to cheaper solar cells and higher throughput in fabrication, as well as lower toxicity per unit cell area. In addition, for tandems with higher perovskite bandgaps, which have the largest potential tandem efficiency, and which have applications in photoelectrochemical splitting of water because of their high voltage, ${ }^{29,30}$ for example, spectrum splitting has significant potential to enhance the efficiency. In the following, we show for which conditions a spectral splitter in a $2 \mathrm{~T}$ perovskite/Si tandem cell is beneficial and what efficiency gains can be achieved. We make a distinction between planar spectral splitters with specular reflectivity, and spectral splitters with a Lambertian scattering distribution that enhances light trapping in the perovskite top cell.

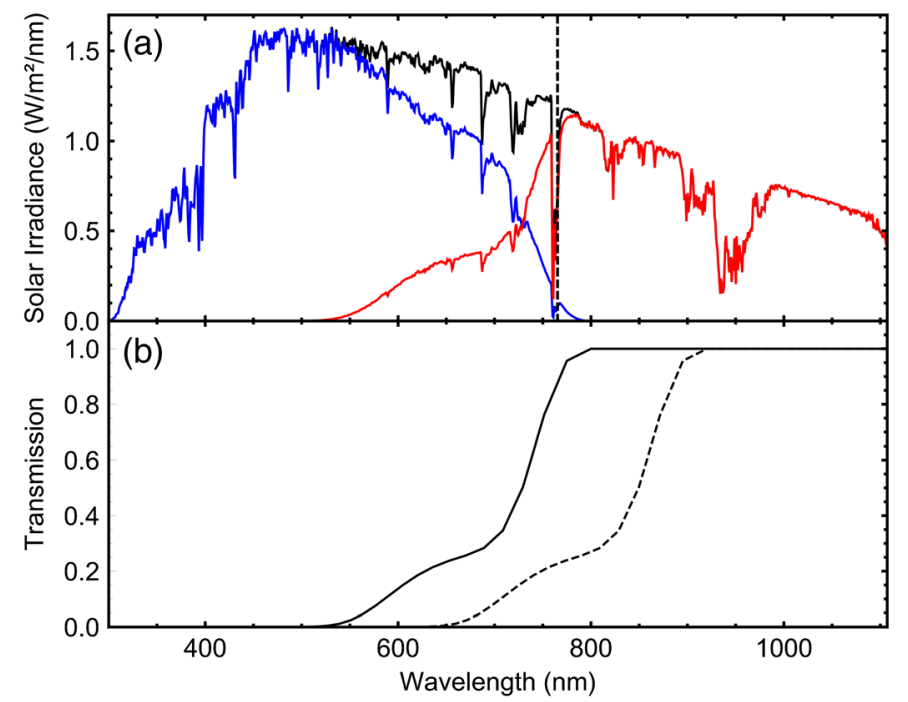

Fig. 1 (a) AM1.5G solar spectrum (black line) with theoretical absorption in 500-nm thick perovskite top cell (blue line) and spectrum directed into Si bottom cell (red line). The vertical dashed line marks the position of the bandgap $E_{\mathrm{BG}}=1.62 \mathrm{eV}$. (b) Transmission spectrum of 500-nm thick perovskite with $E_{\mathrm{BG}}=1.62 \mathrm{eV}$ (solid black line) from Ref. 21 and modeled transmission for 500-nm thick perovskite with $E_{\mathrm{BG}}=1.4 \mathrm{eV}$ (dashed black line). 


\section{Methods}

Throughout the article, we use detailed-balance calculations ${ }^{31}$ to determine the theoretical maximum efficiencies of the different $2 \mathrm{~T}$ tandem configurations. These calculations are based on balancing the bandgap dependent absorption flux (light generated current from the AM1.5G spectrum) with the bandgap and voltage-dependent emission flux (via Planck's equation). From this, current-voltage curves can be constructed. Auger recombination in Si and other nonradiative processes are not taken into account and we assume full current collection from light that is absorbed in the top and bottom cells, meaning ideal electronic properties. While such theoretical maximum efficiencies cannot be reached in reality, they allow a more systematic comparison between different configurations. Similar trends as we find here will also apply for tandem designs in realistic experimental geometries, as we will discuss further on. We differentiate between three cases of light absorption in the perovskite top cell:

1. Full absorption up to the bandgap as is typically done in detailed-balance limit calculations;

2. Ideal current splitting (CS) that assumes that the top cell absorbs exactly the amount of light such that half of the maximum possible current of the bottom cell (as a singlejunction cell) is generated in the top cell;

3. Single pass absorption of a semitransparent top cell with finite thickness $d$ :

$$
A_{\mathrm{TTC}}=(1-\exp (-\alpha \cdot d)),
$$

where $\alpha$ is the absorption coefficient for the transmitting top cell (TTC). To model the bandgap-dependent absorption in the perovskite top cell, we use the wavelengthdependent absorption coefficient of CsFAPbIBr with a bandgap of $E_{\mathrm{BG}}=1.62 \mathrm{eV},{ }^{21}$ which is in the bandgap range of the most frequently used perovskite top cells. Figure 1(b) shows the transmission of a 500-nm-thick layer of this material (black solid line). The transmission for perovskites with the same thickness but different bandgap was then modeled by shifting the graph by the bandgap shift [Fig. 1(b)], similarly to Ref. 32. Our simplified model of light absorption might not fully reflect transmission for specific perovskites with different material compositions and bandgaps; however, it allows us to systematically compare the effect of transparency of the top cell for different bandgaps.

\section{2T Tandem Efficiencies with Semitransparent Top Cells}

The limiting efficiency for $2 \mathrm{~T}$ tandems, assuming perfect absorption up to the bandgap in the top cell of the perovskite/Si tandem, is maximal for $E_{\mathrm{BG}}=1.73 \mathrm{eV}$ with an efficiency of $45.1 \%$ (Fig. 2). This limit strictly adheres to the assumptions that are made for the single-junction detailed balance limit and will be referred to as infinite (top cell) thickness (IT) limit. The limiting efficiency gradually decreases as the top-cell bandgap approaches the Si bandgap (1.12 eV), where all light up to that energy is absorbed in the top cell with no current left for the bottom cell. We then define the CS limit as a condition, for a given perovskite bandgap, where the top cell is not fully absorbing up to its bandgap, but rather absorbs an optimized smaller fraction to obtain current matching (Fig. 2). In this ideal case, the top cell absorbs light such that exactly half of the maximum possible current of the bottom cell (as a single-junction cell) is generated in the top cell. This requires a top cell with a bandgap below $1.73 \mathrm{eV}$. For bandgaps below $1.73 \mathrm{eV}$, the CS limit is well above that for the IT limit of fully absorbing top cells. For higher perovskite bandgaps, full absorption in the top cell is always optimal and equal to the CS limit.

As described above, for any perovskite bandgap the optimized absorption, or equivalently transmission, of the top cells can be realized by selecting the proper top cell thickness (see Sec. 2). We calculate the detailed-balance limiting efficiency using the modeled transmission Eq. (1) for thicknesses in the range 250 to $1000 \mathrm{~nm}$ as a function of bandgap of the top cell (Fig. 2). Several trends can be observed in this figure. First, the shape of every individual TTC limit is similar to the shape of the IT limit, with a pronounced maximum efficiency that is quickly 


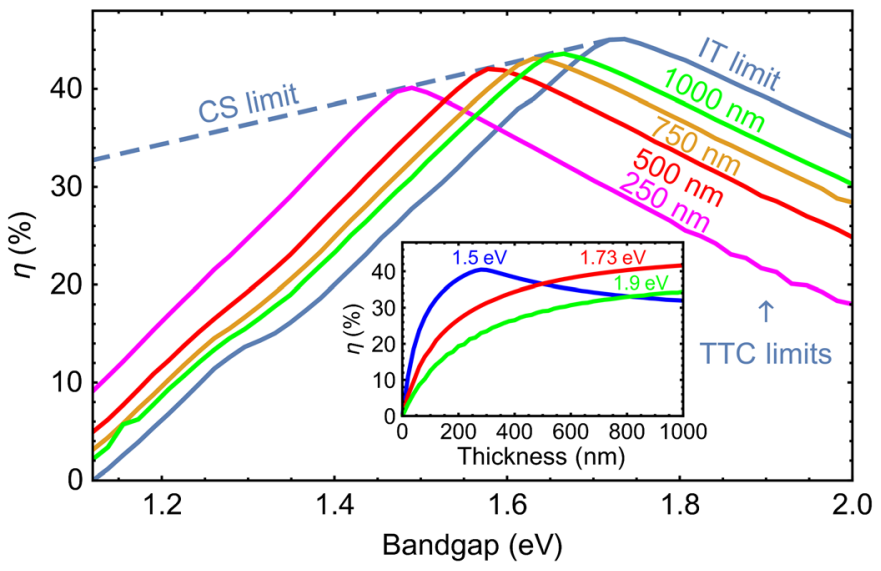

Fig. 2 Detailed-balance efficiency limits for 2T tandem solar cells with Si bottom cell: infinite (top cell) thickness limit (IT) assuming perfect absorption in the top cell (solid blue line); CS limit assuming ideal splitting of the spectrum for perfect current matching in top and bottom cell (dashed blue line); TTC limit assuming realistic absorption/transmission in a perovskite top cell for top cell thickness in the range 250 to $1000 \mathrm{~nm}$. Inset: Comparison of dependence of efficiency on top cell thickness for three different perovskite top cell materials with $E_{\mathrm{BG}}=1.5 \mathrm{eV}, E_{\mathrm{BG}}=1.73 \mathrm{eV}$, and $E_{\mathrm{BG}}=1.9 \mathrm{eV}$.

decreasing for lower or higher bandgaps. This is because of the large sensitivity of $2 \mathrm{~T}$ tandem cells to unbalanced currents in the top and bottom cells. For bandgaps below $1.73 \mathrm{eV}$, the maximum efficiency for every thickness is much higher than the one derived from the IT limit assuming full absorption. The maximum of all four graphs for different thicknesses match the CS curve at the bandgaps where the currents are matched.

Figure 2 shows there is a wide range of perovskite bandgaps $(1.5$ to $1.7 \mathrm{eV})$ for which very high $2 \mathrm{~T}$ tandem efficiencies can be reached by optimizing the perovskite thickness in the practical range of 250 to $1000 \mathrm{~nm}$. For top cells with bandgaps higher than $E_{\mathrm{BG}}=1.73 \mathrm{eV}$, the TTC limit is much lower than the IT limit, because incomplete absorption in the top cell due to the limited thickness limits the overall current. This is where a spectral splitting light trapping layer could be of special interest. Transmitted light above the bandgap is then reflected and trapped in the top cell, such that the infinite thickness limit could be approached for those bandgaps even with finite top cell thicknesses. This is discussed in detail in the next section. The described trends are shown by the inset in Fig. 2 where the TTC limit versus thickness of the top cell is plotted for three different bandgaps. For a bandgap below $E_{\mathrm{BG}}=1.73 \mathrm{eV}$ an ideal thickness can be chosen to reach the maximum TTC efficiency for that bandgap (e.g., $280 \mathrm{~nm}$ at $1.5 \mathrm{eV}$, see the inset). For bandgaps of $E_{\mathrm{BG}}=1.73 \mathrm{eV}$ and higher, the cell ideally is "infinitely" thick to absorb all light above the bandgap energy.

\section{Planar and Lambertian Spectral Splitter}

Next we discuss the effect of a spectral splitter in between the top and bottom cell of a $2 \mathrm{~T}$ tandem cell. We distinguish between: (1) a planar spectral splitter that reflects the light specularly back to the top cell and creates one extra path for absorption in the top cell; (2) a Lambertian spectral splitter that reflects the light back in a cosine angular fashion such that enhanced light trapping can be achieved in the top cell. Figure 3(a) shows a schematic of both spectral splitter geometries. The reflectivity $R(\lambda)$ of the spectral splitter is defined as a step function with tunable step wavelength $\lambda_{\text {step }}$ and reflectance $r$ :

$$
R(\lambda)=\left\{\begin{array}{l}
0, \lambda>\lambda_{\text {step }} \\
r, \quad \lambda \leq \lambda_{\text {step }}
\end{array}\right.
$$

In both cases, we assume that light that is not reflected is transmitted losslessly to the underlying Si substrate. We add one additional absorption path in the top perovskite cell with length $d$ for the 

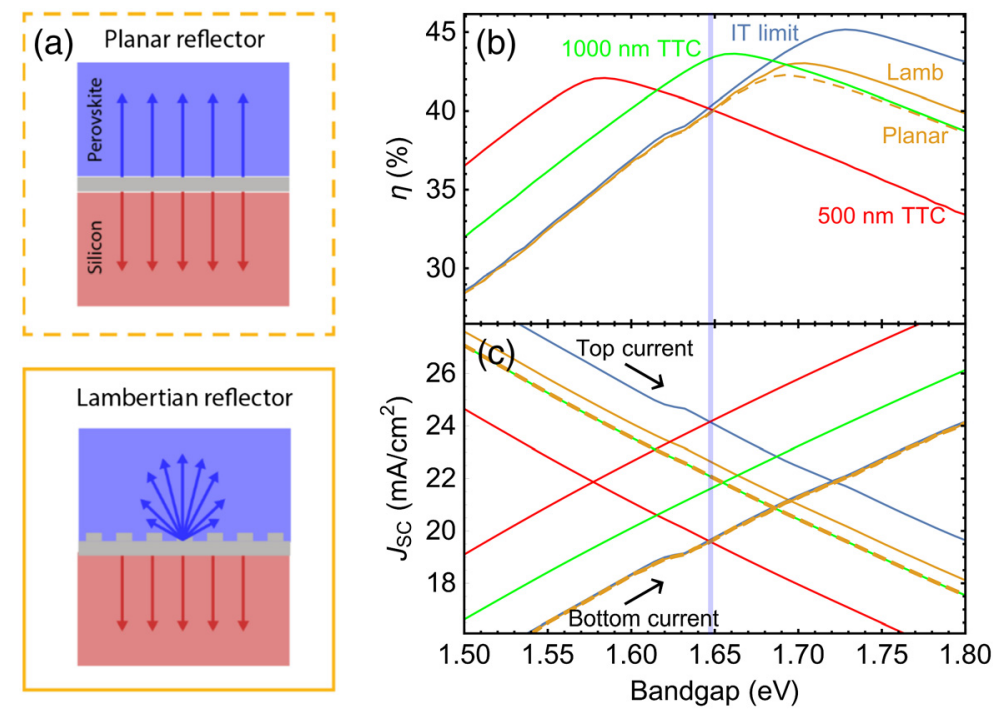

Fig. 3 Spectral splitter in 2T tandem cell. (a) Schematics of planar and Lambertian spectral splitter (in gray). Light is selectively scattered back to the perovskite top cell (blue). The remainder of the spectrum is transmitted to the underlying Si cell (red). (b) IT limiting efficiency for full absorption in the top cell (blue line), TTC limit with 500-nm (red) and 1000-nm (green) thick perovskite top cell. Limiting efficiencies of $2 \mathrm{~T}$ tandems with 500 -nm thick perovskite top cell and planar (dashed yellow line) and Lambertian spectral splitter (solid yellow line). (c) $J_{\mathrm{SC}}$ of top and bottom cell of tandem cells for all limits from (b). The graphs increasing and decreasing with bandgap represent the current of the top and bottom cells, respectively. The bottom cell curves for planar, Lamb, and IT overlay with each other.

planar splitter and length $d_{\mathrm{eff}}=d \cdot \frac{2+x}{1-x}$ with $x=a(\alpha d)^{b}, a=0.935$ and $b=0.67$ to represent the angular distribution scattered by the Lambertian splitter. ${ }^{19,34}$ The absorption in the bottom cell is modified to account for the reflectivity $r$.

First, we assume that the reflectivity is split at the bandgap of the top cell $\left(\lambda_{\text {step }}=\lambda_{\mathrm{BG}}\right)$ and assume $r=1$. In that case for both spectral splitter configurations, the absorption in the Si bottom cell is identical to the one in the infinite thickness limit as no light below the top cell bandgap energy is transmitted to the bottom cell. Figure 3(b) shows the tandem efficiencies for a 500-nm thick perovskite top cell, comparing the TTC limit with the planar and Lambertian spectral splitting limits. Figure 3(c) shows the corresponding top and bottom cell currents. For a 500-nm top cell, the planar or Lambertian spectral splitters strongly improve the tandem efficiency. At $1.70 \mathrm{eV}$, the Lambertian splitter increases the tandem efficiency by more than $5 \%$ to $42.9 \%$. Interestingly, this is even beyond the TTC maximum of $42.0 \%$ that occurs for a bandgap of $1.58 \mathrm{eV}$, reflecting the trend that the higher the absorption in the top cell, the closer the ultimate IT limit that assumes full absorption, which occurs at $1.73 \mathrm{eV}$, is achieved.

To further illustrate the benefit of the spectrum splitters, we show the TTC limit of a 1000-nm top cell in the same figure and compare the short-circuit current $J_{\mathrm{SC}}$ of the top and bottom cells in the lower panel. The top cell $J_{\mathrm{SC}}$ for the $1000-\mathrm{nm}$ thick cell and the $500 \mathrm{~nm}$ cell with planar spectral splitter is identical for all bandgaps as the top cell thickness is effectively doubled by the planar spectral splitter. The bottom cell current, however, is always lower for the 500-nm thick cell with spectrum splitter. This is because for the planar geometry above-bandgap light that is not absorbed in the top cell is transmitted into the Si bottom cell, while with the spectral splitter it is reflected and lost from the front side of the top cell. For the larger bandgaps, the efficiencies of the 1000-nm thick cell and the $500 \mathrm{~nm}$ cell with planar spectral splitter become equal as the identical top cell currents are the limiting factor in both tandems.

The benefit of the Lambertian splitter over the planar splitter is twofold: it further enhances absorption in the top cell due to the larger angular scattering range, and, as a result, less light is lost from the front of the cell. Consequently, the top cell current of the cell with Lambertian spectral splitter is consistently higher than that of the planar spectral splitter while the bottom cell current of both cases is equal. 
The bumps in current and efficiency around $1.63 \mathrm{eV}$ that can be observed for the IT limit as well as the planar and Lambertian spectral splitters come from the fact that within the range between 1.60 and $1.63 \mathrm{eV}$ the AM1.5G spectrum contains almost no photons, and hence the change in current within the top and bottom cells is negligible.

\section{Splitting Conditions}

Next, we investigate the influence on the efficiency of an offset $(\Delta E)$ of the step energy from the top cell bandgap energy as well as incomplete reflectance from the spectrum splitter. This is of interest because it has been found that for $4 \mathrm{~T}$ tandem cells, especially for planar reflectors, the escape losses from the front side of the cell for light at energies just above the top cell bandgap can be detrimental for the overall tandem performance. ${ }^{19}$ To avoid this, we shift the reflection spectrum to slightly higher energy, so the nonabsorbed spectral band just above the top-cell bandgap is transmitted to the Si bottom cell. Figures 4(a) and 4(b) show the efficiency gains/losses $\Delta \eta$ for a $500 \mathrm{~nm}$ top cell with a bandgap of $1.7 \mathrm{eV}$ as a function of $\Delta E$ and $r$, with and without planar and Lambertian spectral splitter, respectively. The gain/loss $\Delta \eta$ is calculated from the difference between a cell with spectral splitter and a cell without, meaning only with realistic reflection (5\% to $6 \%$ within relevant range) that arises from the difference in refractive index at the perovskite-Si interface. In both cases (planar/Lambertian), the best result is found for the highest reflectivity of the spectral splitter, but the figure shows that also for nonideal splitters with $r<1$, as they may be made experimentally, large efficiency gains are expected. For the planar splitter, a maximum achievable efficiency enhancement of $4.2 \%$ is found; for the Lambertian one, a gain of $5.1 \%$ is expected. In both cases, the optimum is found for a shift in the reflectance edge by about $20 \mathrm{meV}$ above bandgap of the top cell. The maxima are marked with black stars in Figs. 4(a) and 4(b).

To further study the impact of the reflectance $r$, we calculate the possible efficiency gain as a function of top cell bandgap for a Lambertian spectral splitter with 500-nm top cell thickness [Fig. 4(c)], again in comparison to a tandem cell with realistic perovskite-Si interface reflection. In agreement with what is described above, efficiency gain is observed for the highest top cell bandgaps, while a loss is observed for the lowest gaps. However, we find that variation of $r$ allows further optimization depending on the top-cell bandgap. An interesting subtlety occurs just at the cutoff energy $1.65 \mathrm{eV}$. Contrary to what was observed for the spectral splitter with $r=1$ in Fig. 3(b), also for smaller bandgaps, a spectral splitter geometry can be beneficial if the reflectivity is reduced.

In Fig. 4(c), one can see that also here a Lambertian spectral splitter is beneficial and increases the efficiency by $2.7 \%$ absolute [marked with black star in Fig. 4(c)], if the reflectance is set to be $r=0.65$. In detailed balance calculations, an equal increase can be reached by increasing the thickness of the top cell, however, in practice an increase in thickness leads to losses in the $V_{\mathrm{OC}}$. For very low reflectivity and small bandgaps (around $1.5 \mathrm{eV}$ ), the efficiency gain goes up as well because for these low bandgaps the bottom cell is limiting and for reflectivity values below 0.05 more light is transmitted into the Si bottom cell, compared with the reference that assumes realistic reflection values at the interface. While the combined bandgap and reflection range for this phenomenon is small, such gains could for example be achieved with a graded intermediate layer, effectively acting as an interface AR coating. ${ }^{14}$ However, for larger bandgaps and low reflectivity, exactly this transmission into the Si layer causes efficiency loss, as the Si cell is not current limiting in this case.

So far, we have assumed $r=0$ for energies below the cut-off energy. To investigate how a nonzero value for $r$ affects the overall performance, we plot the efficiency enhancement as a function of the reflectivity above $\left(r_{\text {perovskite }}\right)$ and below $\left(r_{\mathrm{Si}}\right)$ the energy cut-off for a planar case compared with realistic interface reflection [Fig. 4(d)]. For small $r_{\mathrm{Si}}, r_{\text {perovskite }}$ should ideally be as large as possible for highest efficiencies. In this case, each subcell receives most of the light in the relevant spectral range. However, with increasing $r_{\mathrm{Si}}$, the ideal $r_{\text {perovskite }}$ shifts to lower reflectivity values. This can be explained by considering that high $r_{\mathrm{Si}}$ corresponds to current loss in the bottom cell. As soon as the bottom cell is current limiting, the overall tandem current profits from a lower reflectivity above the cut-off energy, as this will mitigate the current losses in the limiting 

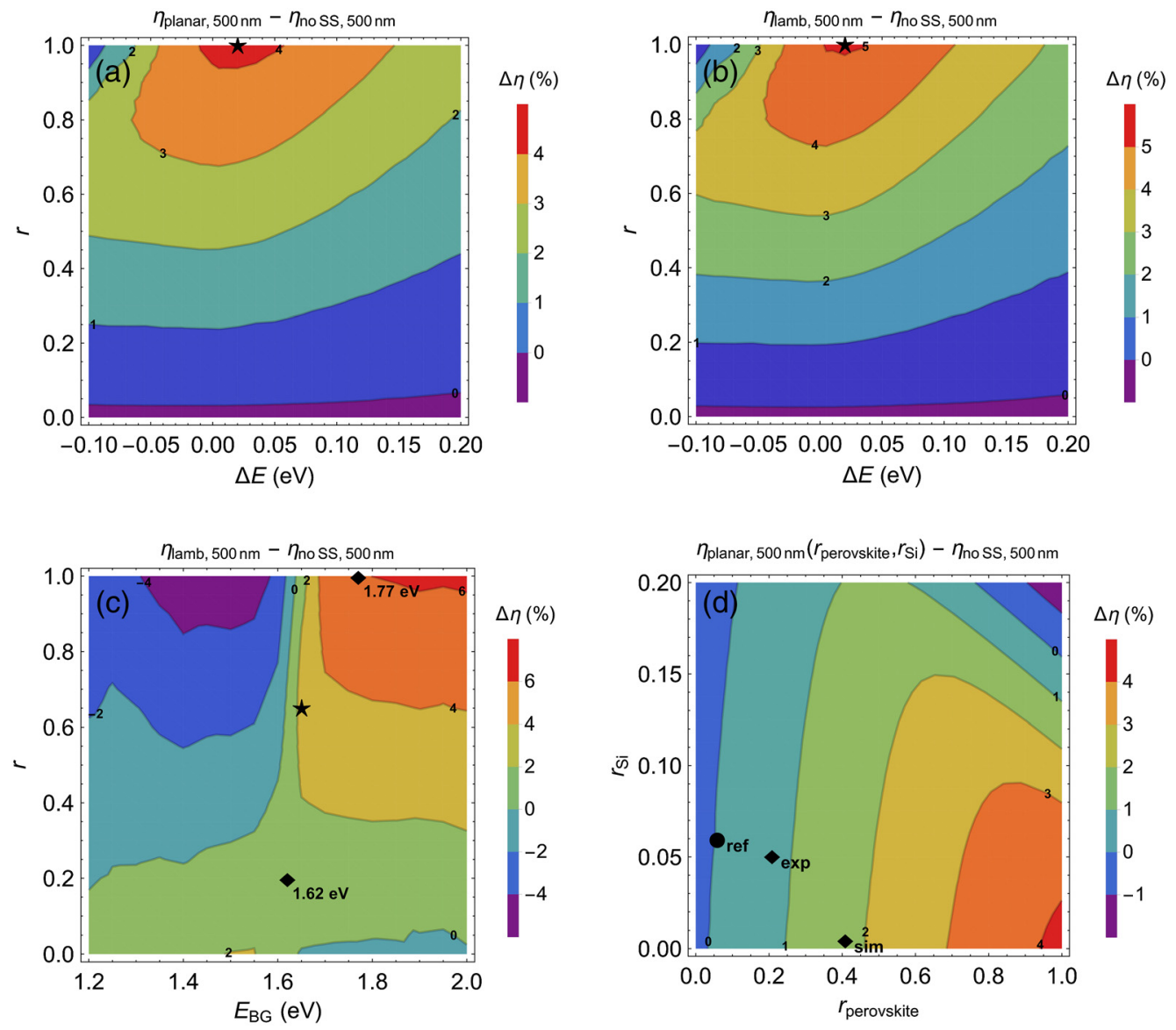

Fig. 4 Absolute efficiency gain of 2T tandem cells depending on reflectance of the spectral splitter, the step wavelength and parasitic absorption in the spectral splitter. Efficiency gain for tandem cells with (a) planar and (b) Lambertian spectral splitter and 500-nm-thick perovskite top cell with $E_{\mathrm{BG}}=1.7 \mathrm{eV}$ compared with TTC limit of the same cell, as function of $\Delta E$ and $r$. The stars in (a) and (b) represent the largest efficiency enhancement. (c) Efficiency gain for tandem cell with Lambertian spectral splitter and 500-nm thick top cell as function of top cell bandgap and reflectance $r$ with $\Delta E=0.02 \mathrm{eV}$. The star indicates the ideal spectral splitting condition for maximal efficiency gain for a tandem cell with a 500-nm-thick perovskite top cell with $E_{\mathrm{BG}}=1.65 \mathrm{eV}$. The diamonds mark the position of maximum gain for two examples of realistic perovskite-silicon cells with $E_{\mathrm{BG}}=1.62 \mathrm{eV}$ and $E_{\mathrm{BG}}=1.77 \mathrm{eV}$. (d) Efficiency gain using a planar spectral splitter as function of reflection above $\left(r_{\text {perovskite }}\right)$ and below $\left(r_{\mathrm{Si}}\right)$ the cutoff energy. The perovskite bandgap is $1.7 \mathrm{eV}$ and $\Delta E=0.02 \mathrm{eV}$. The diamonds mark experimental (exp) and simulated (sim) results for a $4 \mathrm{~T}$ spectral splitter. ${ }^{35}$ The dot (ref) refers to the calculated reflection at a realistic perovskite-Si interface. All figures assume no parasitic absorption.

bottom cell. For example, at $r_{\mathrm{Si}}=0.15$, the ideal $r_{\text {perovskite }}$ is found to be around 0.60 . At this point, the currents in the two subcells are matched. For larger $r_{\text {perovskite }}$ values, the efficiency gain decreases because the bottom cell current decreases, while the top cell current decreases for smaller $r_{\text {perovskite }}$ values. The circular marker in Fig. 4(d) is the calculation assuming realistic reflectivity of a perovskite-Si interface. The diamond-shaped markers indicate values of $r_{\text {perovskite }}$ and $r_{\mathrm{Si}}$ for experimentally realized and simulated 4T spectral splitters, ${ }^{35}$ showing that spectral splitting geometries with these reflectivities can be realized in practice.

We note that all calculations so far were based on an idealized set of assumptions. To get an idea of what efficiency enhancement can be expected in the Lambertian case for realistic materials, we applied detailed-balance calculations using data from selected record cells. ${ }^{36}$ As a first estimate, we took a state-of-the-art perovskite/Si tandem cell with a top-cell bandgap of $1.62 \mathrm{eV}$. The corresponding EQE-spectra and thickness for the top cell layer were used to approximate the absorption coefficient around the bandgap, by assuming unity internal quantum efficiency. 
The resulting absorption coefficient was used to investigate the potential performance of a Lambertian spectral splitter for a 500-nm thick perovskite layer on top of Si. Figure 4(c) contains markers (diamonds) representing this comparison. In the case of the $1.62 \mathrm{eV}$ perovskite, it turns out that an efficiency loss of $0.13 \%$ absolute is to be expected (in the detailed-balance limit) for an ideal reflectance of $r=0.2$.

So far, this analysis assumed detailed-balance-derived open-circuit voltage $\left(V_{\mathrm{OC}}\right)$ and fillfactor $(F F)$ values. To get a more accurate estimate for realistic conditions, we approximate the tandem cell as a nonideal diode under illumination described by Eq. (3):

$$
J(V)=J_{\mathrm{SC}}-J_{0} *\left(\exp \left(\frac{q * V}{n * k * T}\right)-1\right) .
$$

The overall current density $J(V)$ depends on the short-circuit current density $J_{\mathrm{SC}}$ and the voltage-dependent diode current density term with the voltage $V$, the reverse saturation current density $J_{0}$, an ideality factor $n$, Boltzmann's constant $k$, and the temperature $T=298.15 \mathrm{~K}$ (standard test conditions). Using this equation, we can fit the effective recombination current $J_{0, \text { tandem }}$ and ideality factor $n_{\text {tandem }}$ of the full tandem device from the cell's IV-curve. The $V_{\mathrm{OC}}$ was calculated using the $J_{\mathrm{SC}}$ for the Lambertian case as derived above in the detailedbalance calculations, plugging it into the nonideal diode equation and solving the equation for $J(V)=0$. Furthermore, we account for the local $F F$ minimum around the current matching condition ${ }^{37}$ by applying the same relative $F F$-loss that is observed in the detailed-balance analysis above on the listed $F F$ value of the experimental cell. This comparison yields $0.17 \%$ absolute efficiency loss $(r=0.2)$, just slightly lower compared with the initial estimate that was based on only adjusting the absorption coefficient to the experimental value.

To get an estimate for a tandem cell with a higher perovskite bandgap, we take the top cell from the current perovskite/perovskite record tandem cell $(1.77 \mathrm{eV})^{36}$ and determine the absorption coefficient. In this case, due to the nature of the available data, we are limited to an estimation according to the first method used above. For the imagined case of such a top cell on top of a Si bottom cell, this analysis yields an efficiency enhancement of $2.7 \%$ absolute at a reflectance of $r=1$, highlighting that the significance of a Lambertian spectral splitter increases with the top cell bandgap.

We note that the efficiency gains according to this estimate are less than what Fig. 4(c) suggests (diamonds). This is attributed to the fact that for the comparison with the record devices we derived the absorption coefficient from stated $E Q E$ spectra. This procedure tends to overestimate the absorption coefficient, as it also includes absorption beyond the first pass. Hence, the absorption coefficient shows a sharper onset, which weakens gains from spectrum splitting layers. This is a fact that should be kept in mind. For absorption coefficients that strongly deviate in slope (either less or more direct bandgaps), the optimal bandgap values shift but the associated conclusions and trends do not. Overall, this analysis underlines how much more relevant a spectral splitter is for large bandgap perovskite top cell than for small bandgaps.

Finally, an important parameter in the experimental realization of a spectral splitter is the losses that such a layer could introduce into the system. Figure 5 shows the efficiency gain/loss as a function of parasitic absorption, assuming spectrally flat parasitic absorption for a 500-nmthick cell at the perovskite-Si interface. We find that for a bandgap of $1.7 \mathrm{eV}$ and $\Delta E=$ $0.02 \mathrm{meV}$, the range for which a planar/Lambertian spectral splitter is still beneficial strongly depends on the reflection above the bandgap. To understand this, we need to remind ourselves that due to the placement of the spectrum splitter, all light that passes into the bottom cell will encounter it, but the light that is absorbed within the first pass inside the top cell will not. In the case of the top cell, only light that has been scattered back by the spectrum splitter will be affected by parasitic absorption. This means that the current in the bottom cell is linearly dependent on the parasitic absorption, while in the top cell only the fraction of the current that is generated due to reflection from the spectral splitter decreases linearly with parasitic absorption. Thus, when the top cell is current limiting, the dependence of the efficiency gain on parasitic absorption is much weaker compared with cases with a current limiting bottom cell. With increasing parasitic absorption, the loss in the bottom cell increases more rapidly, which means that in the limit of very high parasitic absorption, the bottom cell will always be the current 


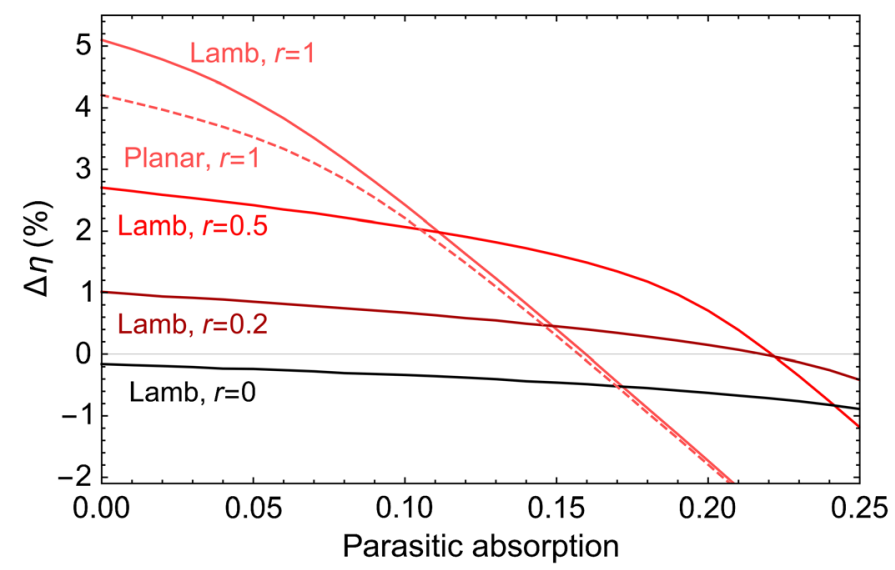

Fig. 5 Efficiency gain for a 2T perovskite/Si tandem cell with Lambertian (solid lines) and planar (dashed pink line) spectral splitter and 500-nm thick top cell, as function of parasitic absorption of the spectral splitter. The efficiency gains are with respect to a cell with realistic interface reflection and no parasitic absorption. $\Delta E=0.02 \mathrm{eV}, E_{\mathrm{BG}}=1.7 \mathrm{eV}$, and $r$ refers to reflection above the cutoff energy. The Lambertian case was plotted for different reflectivity values above the bandgap.

limiting cell. For a bandgap of $1.7 \mathrm{eV}$, we find the interesting case that for maximum reflection $(r=1)$ the top and bottom cells are roughly current matched, while for lower values of $r$ the top cell is limiting and hence we see a shallower slope with decreasing $r$. The initial slope at $r=0$ is non-zero because current losses in the nonlimiting subcell still affect the subcell's $V_{\mathrm{OC}}$ and $F F$ and hence contribute to a slight loss in performance.

\section{Realization}

Considering the theoretical analysis above, and the estimates for what a realistic device with integrated spectral splitter could achieve, we will now give an outline of potential pathways toward realizing such a structure. One important property the spectral splitter needs to have is a sharp step in reflection at the desired cut-off energy, close to the perovskite bandgap. Such steps can be achieved using periodic nanostructures and have been shown for 4T SS devices, ${ }^{35}$ while other concepts, such as graded index interlayers ${ }^{14}$ exhibit broadband features and hence cannot deliver step function-like reflection. While a Bragg reflector can enable a stepwise reflection as well, it can be difficult to retain the electronic performance of the cell and introducing Lambertian scattering from the interface is not possible with such a design. Furthermore, for the optical performance of nanopatterns, it is desirable that the materials that form such a layer exhibit a large contrast in refractive index. Lastly, the integration of a spectral splitter in a $2 \mathrm{~T}$ device requires careful assessment of the influence of used materials on the electronic performance of the cell, and hence two distinct pathways could be explored: fabrication by reshaping the perovskite-Si interface, or introduction of a new, electronically inert material to the interface region.

In the first case, we envision patterning an element of the interface, be it $\mathrm{Si}$, perovskite, or another incorporated layer. Substrate conformal imprint lithography ${ }^{38}$ could be used to transfer a nanopattern onto the cell. From this point, using material removal techniques, such as reactive ion etching, wet-etching, or lift-off in combination with deposition techniques, such as spincoating, evaporation, and sputtering, the chosen material can be patterned. A specific example could for example be the patterning of a specific shape, such as in Ref. 35 into $\mathrm{Si}$, and following this up by spin-coating perovskite on top, to surround these shapes. Taking into account the refractive index of perovskite as the surrounding medium, this could lead to similar resonances as shown previously for $\mathrm{Si}$ features on top of $\mathrm{Si}^{39}$ and these can then be tailored into a spectral splitter layer with desired reflection features. From an electronic perspective, increasing the surface area of Si may lead to higher surface recombination.

In the second pathway, we envision the introduction of a dielectric material that can passivate $\mathrm{Si}$ well, such as $\mathrm{SiN}$ or $\mathrm{Al}_{2} \mathrm{O}_{3}$, providing refractive index contrast and passivation at the same 
time. Provided that band-alignment and surface charge effects do not interfere with the migration of charges through the tandem cell, this could be another option of patterning the Si-perovskite interface. Other materials and patternable regions, such as the backside of the perovskite absorber, can also be considered, provided they do not introduce too large additional electronic losses at the interface. We also note that any pattern at the Si-perovskite interface can translate into a patterned front surface if the top layer is deposited conformally. In principle, this can contribute to enhanced incoupling of light via mechanisms such as Mie-scattering, as is discussed in Ref. 40.

These two pathways involve one to two steps for the creation of a patterned interface. For an efficiency gain of $2.7 \%$, as calculated for large band gap perovskites, this corresponds to a range of $1.3 \%$ to $2.7 \%$ efficiency gain per fabricated layer. For a range between $0.75 \%$ and $1.33 \%$ efficiency gain per perovskite top cell layer that is added to a silicon bottom cell, Werner et al. ${ }^{41}$ estimated manufacturing costs between 46.2 and $19.5 € \mathrm{cts} / \mathrm{W}$, respectively. The referenced range for c-Si modules goes from 25 to $30 € \mathrm{cts} / \mathrm{W}$, which shows that perovskite/Si tandems can be economically interesting. Assuming similar costs for the fabrication steps, and no significant loss in spectrum splitting performance with upscaling, the efficiency gains per fabricated layer found in this work lie within an economically competitive range.

\section{Conclusion}

In this work, we derive the detailed-balance efficiency limit of two-terminal tandem solar cells with a perovskite top cell and a Si bottom cell, considering realistic incomplete absorption conditions for the perovskite top cell. We calculate the theoretically possible efficiency gain due to the introduction of a spectral splitter in between the top and bottom cell. For industrially relevant top cells with bandgaps of $1.7 \mathrm{eV}$ and higher, ${ }^{32}$ a spectral splitter strongly enhances light absorption in the top cell, leading up to $5 \%$ to $6 \%$ absolute efficiency gain in the infinite thickness limit for a 500-nm thick top cell. Using experimental parameters of realistic cells, we predict an efficiency gain for a practical perovskite-tandem cell with Lambertian spectral splitter of $2.7 \%$ for a top cell with a bandgap energy of $1.77 \mathrm{eV}$ and unity spectrum splitter reflectivity. In optimizing the reflectivity of the spectral splitter, we find small subtleties in the 1.6 to $1.7 \mathrm{eV}$ bandgap range, due to unwanted outscattering of light from the front side of the cell. The effect of parasitic absorption that will occur in experimental spectral splitters is also derived. Overall, our work shows there is a bright perspective for the integration of spectral splitters in perovskite-Si tandem solar cells, even if unity reflectivity cannot be achieved experimentally.

\section{Acknowledgments}

This work was part of the research program of the Dutch Research Council (NWO).

\section{References}

1. IRENA, Renewable Energy Capacity Highlights 31 March 2020, pp. 1-3, IRENA (2020).

2. International Renewable Energy Agency, IRENA (2019), Global Energy Transformation: A Roadmap to 2050 (2019).

3. S. Philipps and W. Warmuth, "Photovoltaics report," Fraunhofer ISE, pp. 1-50 (2021).

4. A. Richter, M. Hermle, and S. W. Glunz, "Reassessment of the limiting efficiency for crystalline silicon solar cells," IEEE J. Photovoltaics 3(4), 1184-1191 (2013).

5. A. Al-Ashouri et al., "Monolithic perovskite/silicon tandem solar cell with $>29 \%$ efficiency by enhanced hole extraction," Science (80-.) 370(6522), 1300-1309 (2020).

6. K. Yoshikawa et al., "Silicon heterojunction solar cell with interdigitated back contacts for a photoconversion efficiency over 26\%," Nat. Energy 2(5) (2017).

7. Q. Xu, Y. Zhao, and X. Zhang, "Light management in monolithic perovskite/silicon tandem solar cells," Solar RRL 4(2), 1-16 (2020).

8. D. A. Jacobs et al., "Light management: a key concept in high-efficiency perovskite/silicon tandem photovoltaics," J. Phys. Chem. Lett. 10(11), 3159-3170 (2019). 
9. H. Shen et al., "Monolithic perovskite/Si tandem solar cells: pathways to over $30 \%$ efficiency," Adv. Energy Mater. 10(13), 1902840 (2020).

10. D. T. Grant et al., "Design guidelines for perovskite/silicon 2-terminal tandem solar cells: an optical study," Opt. Express 24(22), A1454 (2016).

11. R. Santbergen et al., "Minimizing optical losses in monolithic perovskite/c-Si tandem solar cells with a flat top cell," Opt. Express 24(18), A1288 (2016).

12. A. Martins et al., "Photonic intermediate structures for perovskite/c-silicon four terminal tandem solar cells," IEEE J. Photovoltaics 7(5), 1190-1196 (2017).

13. D. T. Grant et al., "Towards optical optimization of planar monolithic perovskite/siliconheterojunction tandem solar cells," J. Opt. 18, 064012 (2016).

14. L. Mazzarella et al., "Infrared light management using a nanocrystalline silicon oxide interlayer in monolithic perovskite/silicon heterojunction tandem solar cells with efficiency above 25\%," Adv. Energy Mater. 9(14), 1803241 (2019).

15. M. Chapa et al., "All-thin-film perovskite/C-Si four-terminal tandems: interlayer and intermediate contacts optimization," ACS Appl. Energy Mater. 2(6), 3979-3985 (2019).

16. M. Schultes et al., "Sputtered transparent electrodes (IO:H and IZO) with low parasitic nearinfrared absorption for perovskite- $\mathrm{Cu}(\mathrm{In}, \mathrm{Ga}) \mathrm{Se}_{2}$ tandem solar cells," ACS Appl. Energy Mater. 2(11), 7823-7831 (2019).

17. M. Jošt et al., "Textured interfaces in monolithic perovskite/silicon tandem solar cells: advanced light management for improved efficiency and energy yield," Energy Environ. Sci. 11(12), 3511-3523 (2018).

18. F. Sahli et al., "Fully textured monolithic perovskite/silicon tandem solar cells with $25.2 \%$ power conversion efficiency," Nat. Mater. 17(9), 820-826 (2018).

19. N. N. Lal, T. P. White, and K. R. Catchpole, "Optics and light trapping for tandem solar cells on silicon," IEEE J. Photovoltaics 4(6), 1380-1386 (2014).

20. K. Jäger et al., "Prospects of light management in perovskite/silicon tandem solar cells," Nanophotonics 10, 20200674 (2021).

21. J. Je're' et al., "Complex refractive indices of cesium: formamidinium-based mixed-halide perovskites with optical band gaps from 1.5 to $1.8 \mathrm{eV}$," ACS Energy Lett. 3, 742-747 (2018).

22. A. Hoffmann et al., "Advancing tandem solar cells by spectrally selective multilayer intermediate reflectors," Opt. Express 22(S5), A1270 (2014).

23. K. Bittkau, T. Kirchartz, and U. Rau, "Optical design of spectrally selective interlayers for perovskite/silicon heterojunction tandem solar cells," Opt. Express 26(18), A750 (2018).

24. P. G. O'Brien et al., "Photonic crystal intermediate reflectors for micromorph solar cells: a comparative study," Opt. Express 18(5), 4478 (2010).

25. P. G. O'Brien et al., "Selectively transparent and conducting photonic crystal solar spectrum splitters made of alternating sputtered indium-tin oxide and spin-coated silica nanoparticle layers for enhanced photovoltaics," Sol. Energy Mater. Sol. Cells 102, 173-183 (2012).

26. J. Üpping et al., "Three-dimensional photonic crystal intermediate reflectors for enhanced light-trapping in tandem solar cells," Adv. Mater. 23(34), 3896-3900 (2011).

27. A. Bielawny et al., "3D photonic crystal intermediate reflector for micromorph thin-film tandem solar cell," Phys. Status Solidi Appl. Mater. Sci. 205(12), 2796-2810 (2008).

28. S. Fahr, C. Rockstuhl, and F. Lederer, "Sandwiching intermediate reflectors in tandem solar cells for improved photon management," Appl. Phys. Lett. 101(13), 133904 (2012).

29. J. Jia et al., "Solar water splitting by photovoltaic-electrolysis with a solar-to-hydrogen efficiency over 30\%," Nat. Commun. 7(May), 13237 (2016).

30. M. G. Walter et al., "Solar water splitting cells," Chem. Rev. 110(11), 6446-6473 (2010).

31. A. De Vos, "Detailed balance limit of the efficiency of tandem solar cells," J. Phys. D. Appl. Phys. 13(5), 839-846 (1980).

32. E. Köhnen et al., " $27.9 \%$ efficient monolithic perovskite/silicon tandem solar cells on industry compatible bottom cells," Solar RRL 5, 2100244 (2021).

33. S. Rühle, "The detailed balance limit of perovskite/silicon and perovskite/CdTe tandem solar cells," Phys. Status Solidi Appl. Mater. Sci. 214(5), 1600955 (2017).

34. M. A. Green, "Lambertian light trapping in textured solar cells and light-emitting diodes: analytical solutions," Prog. Photovoltaics Res. Appl. 10(4), 235-241 (2002). 
35. V. Neder et al., "Four-terminal perovskite/silicon tandem solar cell with integrated Mieresonant spectral splitter metagrating," arXiv, arXiv:2012.12649v1, pp. 1-4 (2020).

36. M. A. Green et al., "Solar cell efficiency tables (version 56)," Prog. Photovoltaics Res. Appl. 28(7), 629-638 (2020).

37. C. Ulbrich et al., "Matching of silicon thin-film tandem solar cells for maximum power output," Int. J. Photoenergy 2013, 314097 (2013).

38. M. Verschuuren et al. "Large area nanoimprint by substrate conformal imprint lithography (SCIL)," Adv. Opt. Technol. 6(3-4), 243-264 (2017).

39. J. van de Groep et al., "Designing dielectric resonators on substrates: combining magnetic and electric resonances," Opt. Express 21, 26285-26302 (2013).

40. V. E. Ferry et al., "Improved red-response in thin film a-Si:H solar cells with soft-imprinted plasmonic back reflectors," Appl. Phys. Lett. 95, 183503 (2009).

41. J. Werner et al., "Perovskite/silicon tandem solar cells: marriage of convenience or true love story? An overview," Adv. Mater. Interfaces 5, 1700731 (2018).

Biographies of the authors are not available. 